\title{
Elaboração da versão em Português Brasileiro do teste de identificação de sentenças dicóticas (DSI)
}

\author{
Development of the Brazilian Portuguese version of the \\ dichotic sentence identification test (DSI)
}

\author{
Adriana Neves de Andrade ${ }^{1}$, Daniela Gil², Maria Cecília Martinelli Iório ${ }^{3}$
}

\begin{abstract}
RESUMO
Objetivo: Elaborar a versão em Português Brasileiro do teste de identificação de sentenças dicóticas (DSI). Métodos: As frases utilizadas na versão em Português do teste DSI foram extraídas do teste de identificação de sentenças sintéticas (SSI) em Português. Foi realizado um método de combinação onde formaram-se 45 pares de frases, sendo cada par de frases correspondente a um item de teste. Os itens foram distribuídos em cinco grupos, sendo que cada grupo corresponde a uma condição do teste. Para realizar essa disposição, criou-se um programa na plataforma computacional Matlab 7.0, que utilizou o método Monte Carlo ou método da força bruta para obter a distribuição dos itens em cada grupo. As frases foram editadas, gravadas e finalizadas; para a manipulação dos dados utilizou-se o programa CaKewalk Sonar 2.2. Para a finalização, acabamento e padronização da gravação utilizou-se o programa Sound Forge. Resultados: Como resultado obteve-se um compact disc com seis faixas para serem aplicadas na avaliação comportamental do processamento auditivo, nas seguintes etapas: calibração, treino, integração binaural, escuta direcionada à direita e escuta direcionada à esquerda. Para o uso na reabilitação dos distúrbios do processamento auditivo recomenda-se o uso das faixas de calibração e de treinamento auditivo. Conclusão: O compact disc com a versão em Português Brasileiro do teste DSI constou de seis faixas: calibração, treino, integração binaural, escuta direcionada à direita, escuta direcionada à esquerda e treinamento auditivo.
\end{abstract}

Descritores: Testes auditivos; Audição; Testes de discriminação da fala; Audiometria da fala; Córtex auditivo

\section{INTRODUÇÃO}

O Processamento Auditivo refere-se à eficiência e à eficácia com a qual o sistema nervoso central utiliza a informação auditiva $^{(1)}$; sua avaliação pode ser realizada com testes auditivos comportamentais (verbais e não verbais) ${ }^{(2)}$ que avaliam o desempenho dos indivíduos em situações similares às da vida cotidiana. Dentre os testes auditivos comportamentais existentes, há o teste de identificação de sentenças dicóticas (DSI - Dichotic Sentence Identification).

$\mathrm{O}$ teste DSI foi desenvolvido para avaliar a função auditi-

Trabalho realizado no Núcleo Integrado de Assistência, Pesquisa e Ensino em Audição - NIAPEA - da Disciplina dos Distúrbios da Audição do Departamento de Fonoaudiologia da Universidade Federal de São Paulo - UNIFESP - São Paulo (SP), Brasil.

(1) Mestre, Pós-graduanda (Doutorado) em Ciências pelo programa de Distúrbios da Comunicação Humana pela Universidade Federal de São Paulo - UNIFESP - São Paulo (SP), Brasil.

(2) Doutora, Professora Adjunto do Departamento de Fonoaudiologia da Universidade Federal de São Paulo - UNIFESP - São Paulo (SP), Brasil.

(3) Livre-docente, Professora Associada do Departamento de Fonoaudiologia da Universidade Federal de São Paulo - UNIFESP - São Paulo (SP), Brasil. Endereço para correspondência: Adriana Neves de Andrade. R. São Francisco, 498, Santo Antonio, São Caetano do Sul (SP), Brasil, CEP: 09530-050. E-mail: adriandrad@hotmail.com

Recebido em: 27/9/2009; Aceito em: 14/3/2010 va central em indivíduos com perda auditiva periférica ${ }^{(3)}$. As sentenças utilizadas no teste DSI foram selecionadas a partir do teste de identificação de sentenças sintéticas (Synthetic Sentece Identification - SSI) ${ }^{(4)}$. A versão clínica do DSI é constituída de 30 pares de sentenças apresentadas dicoticamente a $50 \mathrm{~dB}$ NS ou no nível de escuta mais confortável referido pelo paciente. $\mathrm{O}$ indivíduo avaliado deve identificar as duas sentenças apresentadas auditivamente em uma lista impressa com seis frases. Esse instrumento é utilizado para avaliar a habilidade auditiva de integração binaural, sendo um teste com sobrecarga linguística e com redundância, podendo ser aplicado em indivíduos com perda auditiva ${ }^{(5)}$.

No Brasil, nos últimos anos, a avaliação de indivíduos com alteração auditiva periférica aumentou ${ }^{(6-9)}$, ampliando também a necessidade de elaborar instrumentos específicos para essa população. Neste panorama, esta pesquisa teve como objetivo elaborar a versão em Português Brasileiro do teste DSI.

\section{MÉTODOS}

Este estudo foi analisado e aprovado pelo Comitê de Ética em Pesquisa da Universidade Federal de São Paulo sob o n0322/07, e realizado no Núcleo Integrado de Assistência, Pesquisa e Ensino em Audição (NIAPEA) da Disciplina de 
Distúrbios da Audição do Departamento de Fonoaudiologia da Universidade Federal de São Paulo, entre janeiro e setembro de 2008.

Na versão para o Português, para a elaboração do teste DSI, selecionou-se as frases extraídas do teste SSI em Português Brasileiro $^{(10)}$ (a partir do CD que é parte do manual de avaliação do processamento auditivo central) ${ }^{(11)}$ e realizou-se um procedimento de combinação. As dez frases foram agrupadas duas a duas, com a condição de não surgirem apresentações consecutivas em canais opostos, não havendo preocupação com a ordem de aparecimento das frases em cada par. O número de combinações possíveis pôde ser expresso pela equação a seguir:

$C_{n, c}=\frac{n !}{(n-c) ! c !}$

Onde, $C_{n, c}$ possíveis combinações; $n$ total de elementos e $c$ elementos por grupo. Obteve-se, portanto:

$C_{10,2}=\frac{10 !}{(10-2) ! 2 !} \quad C_{10,2}=45$

A partir da equação, foi possível, a formação de 45 pares distintos de frases. As frases foram classificadas com números de 1 a 10, com o objetivo de facilitar a apresentação dos agrupamentos (Quadro 1).

Para elaboração do DSI, as dez frases foram agrupadas em quatro grupos de dez e um grupo de cinco pares. A disposição dos pares em cada grupo teve que satisfazer as seguintes restrições:

- cada frase deveria ser apresentada apenas uma vez, em cada lado, para cada grupo;

- $\quad$ a ordem de apresentação das frases, para cada lado, deveria ser aleatória;

- deveria haver um espaçamento de pelo menos uma apresentação entre a apresentação da mesma frase entre os lados.

Para realizar essa disposição, criou-se um programa na plataforma Matlab 7.0, que utilizou o método Monte Carlo ou método da força bruta para obter a distribuição dos pares em cada grupo. Com estes instrumentos, foi possível realizar sorteios aleatórios que apresentaram uma distribuição normal, definir as frases para cada lado em cada grupo, após uma série de testes que enquadraram as restrições apresentadas e, como
Quadro 1. Numeração das frases extraídas do Teste de Identificação de Sentenças Sintéticas

\begin{tabular}{|l|l|}
\hline Número & Frase \\
\hline 1 & Que ignora o fim principal é ganhar \\
\hline 2 & A porta larga para ser mais rápido \\
\hline 3 & Gosta muito crer te dá muito para \\
\hline 4 & Quarto golpe de estado e o campo \\
\hline 5 & Sobre minha cabeça está de Deus pai \\
\hline 6 & Ação humilde é bem claro o céu \\
\hline 7 & Confiança em minha alma cai dentro de \\
\hline 8 & Sempre e corre muito mais bonito que \\
\hline 9 & Grande general chega já e não creias \\
\hline 10 & Assista a aula de papel branco na. \\
\hline
\end{tabular}

resultado, formar os quarenta e cinco pares que foram divididos em cinco grupos (Quadro 2).

Após obter os cinco grupos, realizou-se em estúdio a gravação do DSI. Para facilitar a gravação do teste, as frases foram colocadas após os números em algarismos arábicos nos quarenta e cinco pares de frases obtidos por meio do método de combinação. A gravação do compact disc foi realizada no AM Áudio Stúdio. Para a manipulação dos dados utilizou-se o programa CaKewalk Sonar 2.2, com este programa, a faixa do teste SSI foi digitalmente extraída e editada do compact disc original.

Após a edição, os canais referentes às frases e à história, foram separados. O canal referente à história do Brasil utilizado no teste SSI, foi descartado e o canal referente às frases foi transformado em uma faixa alvo monocanal.

$\mathrm{Na}$ faixa alvo, foi necessário utilizar o recurso noisegate para retirar o ruído da gravação original e também aumentar em seis decibels a intensidade das frases para permitir que a voz do locutor fosse mais audível. Estes ajustes foram realizados sem interferir na qualidade da gravação original. A partir da faixa alvo, as frases foram editadas e rearranjadas nos dois canais, de acordo com os cinco grupos pré-determinados, formando assim novas faixas.

Na versão em Português do DSI, não foi possível padronizar o tamanho das frases, como no teste em inglês, porque as frases do teste SSI em Português têm extensões que variam de dois a quatro segundos. Só seria possível a edição de todas

Quadro 2. Combinações obtidas a partir do Método Monte Carlo

\begin{tabular}{|c|c|c|c|c|c|c|c|c|c|}
\hline \multicolumn{2}{|c|}{ Grupo 1} & \multicolumn{2}{|c|}{ Grupo 2} & \multicolumn{2}{|c|}{ Grupo 3} & \multicolumn{2}{|c|}{ Grupo 4} & \multicolumn{2}{|c|}{ Grupo 5} \\
\hline OD & $\mathrm{OE}$ & OD & $\mathrm{OE}$ & OD & OE & $O D$ & $\mathrm{OE}$ & OD & $\mathrm{OE}$ \\
\hline 3 & 10 & 7 & 9 & 9 & 1 & 8 & 3 & 1 & 2 \\
\hline 6 & 9 & 10 & 8 & 6 & 10 & 6 & 2 & 5 & 7 \\
\hline 5 & 1 & 2 & 7 & 2 & 9 & 7 & 1 & 6 & 8 \\
\hline 9 & 8 & 6 & 5 & 5 & 8 & 2 & 8 & 9 & 10 \\
\hline 2 & 5 & 8 & 1 & 7 & 3 & 1 & 4 & 3 & 4 \\
\hline 7 & 6 & 9 & 3 & 10 & 2 & 9 & 5 & & \\
\hline 4 & 2 & 1 & 10 & 4 & 7 & 10 & 7 & & \\
\hline 1 & 3 & 4 & 6 & 1 & 6 & 4 & 9 & & \\
\hline 8 & 7 & 3 & 2 & 8 & 4 & 5 & 10 & & \\
\hline 10 & 4 & 5 & 4 & 3 & 5 & 3 & 6 & & \\
\hline
\end{tabular}

Legenda: $\mathrm{OD}=$ orelha direita; $\mathrm{OE}=$ orelha esquerda 
as frases para dois segundos se os intervalos entre os fonemas bem como as extensões das vogais nas frases fossem reduzidas e essa redução comprometeria a inteligibilidade da fala.

$\mathrm{Na}$ formação das novas faixas, o início das frases foi alinhado e utilizou-se intervalos regulares entre as apresentações dos pares de frases, de forma que as distâncias entre os pares obedecessem ao mesmo intervalo de tempo, de acordo com o término da frase mais longa. Para a finalização, acabamento e padronização da gravação utilizou-se o programa Sound Forge.

\section{RESULTADOS}

Terminado o trabalho de edição, como resultado final, obteve-se um compact disc com seis faixas (Anexo 1).

Após a elaboração do compact disc, para a utilização na prática clínica, a cada par de frases foi atribuído um número que corresponde a um item do teste. A partir dessa lista foi criado um protocolo específico (Anexo 2), onde o avaliador deve assinalar certo $(\checkmark)$ ou errado $(\boldsymbol{x})$ no quadrado corresponde ao item e à orelha examinada. Cada acerto ou erro na etapa de treino corresponde a $20 \%$ e nas demais etapas a $10 \%$. Assim, quando um indivíduo acertar todos os itens terá um total de $100 \%$ de acertos para todas as etapas.

\section{DISCUSSÃO}

A versão original do teste $\operatorname{DSI}^{(1)}$ consta de 90 itens, para a etapa de treino são apresentados os 30 últimos itens do teste, sendo que dez pares de sentenças são apresentados em situação monoaural (cinco apenas na orelha direita e cinco na orelha esquerda) e 20 apresentadas dicoticamente (nas orelhas direita e esquerda simultaneamente). Para facilitar a utilização na prática clínica, a versão brasileira do teste DSI, têm uma faixa específica para o treino em que é aplicada apenas a etapa de integração binaural uma vez que essa é a situação de escuta mais difícil do teste.

No teste original em Inglês ${ }^{(3)}$, após o treino, os indivíduos ouvem os 90 itens do teste ( 30 itens em cada etapa: integração binaural, escuta direcionada a direita e escuta direcionada a esquerda) e em todas as condições os sujeitos avaliados devem anotar em uma folha de respostas as frases ouvidas. Como o teste ficou muito extenso, houve a elaboração da versão clínica, na qual, ao invés de utilizar as dez frases do teste SSI, foram utilizadas apenas seis frases apresentadas em 30 itens (dez itens em cada etapa do teste). Essa última versão é comercializada nos Estados Unidos pela Auditec St. Louis.

A adaptação cultural de testes auditivos comportamentais e a gravação do teste por um falante nativo deve ser realizada até mesmo em países que possuem idioma comum, devido às diferenças nos aspectos suprasegmentais da fala.

Na Austrália como o idioma oficial é o Inglês, os audiologistas aplicavam a mesma bateria de testes elaboradas em Inglês, mas observaram que os indivíduos idosos nativos tinham reduzido desempenho nesses testes, então, elaboraram versões australianas para os testes: Staggered Spondaic Word (SSW) ${ }^{(12)}$, Synthetic Sentence Identification $(\mathrm{SSI})^{(4)}$ e Dichotic Sentence Identification (DSI) ${ }^{(3)}$, as versões elaboradas receberam respectivamente os nomes de: Macquarie Staggered Spondaic
Word (MSSW) ${ }^{(13)}$, Macquarie Synthetic Sentence Identification (MSSI) ${ }^{(14)}$ e Macquarie Dichotic Sentence Identification $(\mathrm{MDSI})^{(15)}$. Esses testes são comercializados para a Austrália e Nova Zelândia pela Universidade Macquarie.

$\mathrm{O}$ teste $\mathrm{MDSI}^{(15)}$ foi elaborado a partir das sentenças do teste MSSI ${ }^{(14)}$. As frases foram agrupadas aos pares, randomicamente, sendo que quatro sentenças foram utlizadas para o treino e seis sentenças para o teste. Como resultado, formaramse 30 pares de frases divididas em seis faixas para a aplicação na etapa de integração binaural.

Em relação ao número de sentenças utilizadas na elaboração do teste DSI, a versão em Português Brasileiro assemelha-se à versão original do teste DSI em Inglês ${ }^{(3)}$, pois foram utilizadas as dez sentenças do teste SSI em Português.

No que diz respeito ao número de itens, 90 na versão inglesa $^{(3)}$ e 45 na versão brasileira, essa diferença ocorreu porque na versão inglesa ${ }^{(3)}$ a combinação entre as frases foi realizada duas a duas sem restrições e na versão brasileira as frases foram agrupadas duas a duas, com a condição de não surgirem apresentações consecutivas em canais opostos. Optamos por realizar essa disposição para deixar o teste mais fidedigno, pois se o indivíduo apontar as frases apresentadas podemos inferir que ele ouviu o estímulo e utilizou a habilidade auditiva solicitada no teste. Se não houvesse essa condição, e a mesma frase fosse apresentada consecutivamente, não teríamos como saber se o acerto ocorreu pela a utilização da habilidade auditiva ou da memória.

Com a finalidade de elaborar um material que fosse eficaz e de fácil aplicação na prática clínica, criamos uma faixa para cada etapa do teste a saber: calibração, treino, integração binaural, escuta direcionada à direita, escuta direcionada à esquerda e treinamento auditivo. Foi pensando na avaliação e na reabilitação auditiva que optamos por elaborar uma faixa específica para o treinamento auditivo, assim não se incorre no erro de utilizar o mesmo estímulo para avaliação e treinamento auditivo.

Com este estudo, foi possível elaborar um teste em Português para avaliar a função auditiva central em indivíduos com queixas relacionadas ao processamento auditivo, com a vantagem de poder ser utilizado em portadores de alteração auditiva periférica. Pretende-se assim utilizá-lo posteriormente na avaliação de indivíduos normouvintes para se estabelecer o padrão de normalidade para a população brasileira e em seguida aplicá-lo em uma população com deficiência auditiva neurossensorial.

\section{CONCLUSÃO}

A elaboração da versão em Português Brasileiro do teste DSI, constou de seis faixas a saber: Calibração, Treino, Integração binaural, Escuta Direcionada à Direita, Escuta Direcionada à Esquerda e Treinamento Auditivo.

\section{AGRADECIMENTOS}

Agradecemos à Fundação de Amparo à Pesquisa do Estado de São Paulo (FAPESP), pelo apoio concedido para realização dessa pesquisa, sob processo número 07/51231-1. 


\begin{abstract}
Purpose: To develop the Brazilian Portuguese version of the dichotic sentence idenfication test (DSI). Methods: The sentences used in the Portuguese version of DSI test were extracted from the Synthetic Sentece Identification (SSI) test in Portuguese. A method of combination was used in order to create 45 pairs of sentences, each pair corresponding to a test item. The items were divided into five groups, each group corresponding to a specific test condition. For this purpose, a computer program was designed in the Matlab 7.0 computing platform, using the Monte Carlo method, or the brute force method, for the distribution of items in each group. The sentences were edited, recorded and finalized; for manipulation of data it was used the CaKewalk Sonar 2.2 program. Finally, the Sound Forge program was used for finalization and standardization of the recording. Results: As a result, a compact disc with six tracks was obtained to be used as part of the behavioral assessment of auditory processing, including the following tracks: calibration, practice, binaural integration, directed attention to the right and to the left ears. The use of the calibration and auditory training tracks is recommended for use in the rehabilitation of auditory processing disorders. Conclusion: The compact disc with the Brazilian Portuguese version of the DSI test consisted of six tracks: calibration, practice, binaural integration, directed attention to the right and to the left ears and auditory training.
\end{abstract}

Keywords: Hearing tests; Hearing; Speech discrimination test; Audiometry, speech; Auditory cortex

\section{REFERÊNCIAS}

1. American Speech-Language-Hearing Association (ASHA) (2005) [Internet]. (Central) Auditory Processing Disorders [Technical Report]. [citado 2008 Fev 4] Disponível em: http://www.asha.org/docs/html/ tr2005-00043.html

2. McFarland DJ, Cacace AT. Modality specificity as a criterion for diagnosing central auditory processing disorders. Am J Audiol. 1995;4(1):36-48

3. Fifer RC, Jerger JF, Berlin CI, Tobey EA, Campbell JC. Development of a dichotic sentence identification test for hearing-impaired adults. Ear Hear. 1983;4(6):300-5.

4. Speaks C, Jerger J. Method for measurement of speech identification. J Speech Hear Res.1965;8:185-94.

5. Jerger J, Chmiel R, Allen J, Wilson A. Effects of age and gender on dichotic sentence identification. Ear Hear. 1994;15(4):274-86.

6. Quintero SM, Marotta RMB, Marone SAM. Avaliação do processamento auditivo de indivíduos idosos com e sem presbiacusia por meio do teste de reconhecimento de dissílabos em tarefa dicótica - ssw. Rev Bras Otorrinolaringol. 2002;68(1):28-33.

7. Perrella ACM, Branco-Barreiro FCA. Avaliação da função auditiva central em idosos e suas contribuições para a adaptação de próteses auditivas. Disturb Comunic. 2005;17(3):333-346.
8. Amorim RMC, Almeida K. Estudo do benefício e da aclimatização em novos usuários de próteses auditivas. Pró-Fono. 2007;19(1):39-48.

9. Miranda EC, Andrade AN, Gil D, Iorio MCM. A efetividade do treinamento auditivo formal em idosos usuários de próteses auditivas no período de aclimatização. Rev Soc Bras Fonoaudiol. 2007;12(4):316321.

10. Almeida CIR, Caetano MHU. Logoaudiometria utilizando sentenças sintéticas. Rev Bras Otorrinolaringol. 1988;54(3):68-72.

11. Pereira LD, Schochat E. Processamento auditivo central: manual de avaliação. São Paulo: Lovise; 1997.

12. Katz J. The use of staggered spondaic words for assessing the integrity of the central auditory system. J Aud Res. 1962;2: 327-37.

13. Golding M, Birtles G. Macquarie University Speech Tests. Sydney: The Speech, Hearing and Language Research Centre, Macquarie University; 2001.

14. Golding M. The SSI test and its standardization for Australian use. Sydney: The Speech, Hearing and Language Research Center, Macquarie University; 1983.

15. Golding M. The development of two speech based tests of central auditory function for Australian use. Aust N Z J Audiol. 2001;23(1):1-9. 
Anexo 1. Compact disc da versão em Português Brasileiro do teste de identificação de sentenças dicóticas (DSI)

\begin{tabular}{|c|c|}
\hline \multicolumn{2}{|l|}{ Faixa 1 - Calibração } \\
\hline Canal 1 - Orelha Direita & Canal 2 - Orelha Esquerda \\
\hline Tom puro & Tom puro \\
\hline \multicolumn{2}{|l|}{ Faixa 2 - Treino } \\
\hline Canal 1 - Orelha Direita & Canal 2 - Orelha Esquerda \\
\hline 1 Que ignora o fim principal é ganhar & 2 A porta larga para ser mais rápido \\
\hline 5 Sobre minha cabeça está de Deus pai & 7 Confiança em minha alma cai dentro de \\
\hline 6 Ação humilde é bem claro o céu & 8 Sempre e corre muito mais bonito que \\
\hline 9 Grande general chega já e não creias & 10 Assista a aula de papel branco na. \\
\hline 3 Gosta muito crer te dá muito para & 4 Quarto golpe de estado e o campo \\
\hline \multicolumn{2}{|l|}{ Faixa 3 - Integração Binaural } \\
\hline Canal 1 - Orelha Direita & Canal 2 - Orelha Esquerda \\
\hline 3 Gosta muito crer te dá muito para & 10 Assista a aula de papel branco na. \\
\hline 6 Ação humilde é bem claro o céu & 9 Grande general chega já e não creias \\
\hline 5 Sobre minha cabeça está de Deus pai & 1 Que ignora o fim principal é ganhar \\
\hline 9 Grande general chega já e não creias & 8 Sempre e corre muito mais bonito que \\
\hline 2 A porta larga para ser mais rápido & 5 Sobre minha cabeça está de Deus pai \\
\hline 7 Confiança em minha alma cai dentro de & 6 Ação humilde é bem claro o céu \\
\hline 4 Quarto golpe de estado e o campo & 2 A porta larga para ser mais rápido \\
\hline 1 Que ignora o fim principal é ganhar & 3 Gosta muito crer te dá muito para \\
\hline 8 Sempre e corre muito mais bonito que & 7 Confiança em minha alma cai dentro de \\
\hline 10 Assista a aula de papel branco na & 4 Quarto golpe de estado e o campo \\
\hline \multicolumn{2}{|l|}{ Faixa 4 - Escuta Direcionada à Direita } \\
\hline Canal 1 - Orelha Direita & Canal 2 - Orelha Esquerda \\
\hline 7 Confiança em minha alma cai dentro de & 9 Grande general chega já e não creias \\
\hline 10 Assista a aula de papel branco na. & 8 Sempre e corre muito mais bonito que \\
\hline 2 A porta larga para ser mais rápido & 7 Confiança em minha alma cai dentro de \\
\hline 6 Ação humilde é bem claro o céu & 5 Sobre minha cabeça está de Deus pai \\
\hline 8 Sempre e corre muito mais bonito que & 1 Que ignora o fim principal é ganhar \\
\hline 9 Grande general chega já e não creias & 3 Gosta muito crer te dá muito para \\
\hline 1 Que ignora o fim principal é ganhar & 10 Assista a aula de papel branco na. \\
\hline 4 Quarto golpe de estado e o campo & 6 Ação humilde é bem claro o céu \\
\hline 3 Gosta muito crer te dá muito para & 2 A porta larga para ser mais rápido \\
\hline 5 Sobre minha cabeça está de Deus pai & 4 Quarto golpe de estado e o campo \\
\hline \multicolumn{2}{|l|}{ Faixa 5 - Escuta Direcionada à Esquerda } \\
\hline Canal 1 - Orelha Direita & Canal 2 - Orelha Esquerda \\
\hline 9 Grande general chega já e não creias & 1 Que ignora o fim principal é ganhar \\
\hline 6 Ação humilde é bem claro o céu & 10 Assista a aula de papel branco na \\
\hline 2 A porta larga para ser mais rápido & 9 Grande general chega já e não creias \\
\hline 5 Sobre minha cabeça está de Deus pai & 8 Sempre e corre muito mais bonito que \\
\hline 7 Confiança em minha alma cai dentro de & 3 Gosta muito crer te dá muito para \\
\hline 10 Assista a aula de papel branco na & 2 A porta larga para ser mais rápido \\
\hline 4 Quarto golpe de estado e o campo & 7 Confiança em minha alma cai dentro de \\
\hline 1 Que ignora o fim principal é ganhar & 6 Ação humilde é bem claro o céu \\
\hline 8 Sempre e corre muito mais bonito que & 4 Quarto golpe de estado e o campo \\
\hline 3 Gosta muito crer te dá muito para & 5 Sobre minha cabeça está de Deus pai \\
\hline \multicolumn{2}{|l|}{ Faixa 6 - Treinamento Auditivo } \\
\hline Canal 1 - Orelha Direita & Canal 2 - Orelha Esquerda \\
\hline 8 Sempre e corre muito mais bonito que & 3 Gosta muito crer te dá muito para \\
\hline 6 Ação humilde é bem claro o céu & 2 A porta larga para ser mais rápido \\
\hline 7 Confiança em minha alma cai dentro de & 1 Que ignora o fim principal é ganhar \\
\hline 2 A porta larga para ser mais rápido & 8 Sempre e corre muito mais bonito que \\
\hline 1 Que ignora o fim principal é ganhar & 4 Quarto golpe de estado e o campo \\
\hline 9 Grande general chega já e não creias & 5 Sobre minha cabeça está de Deus pai \\
\hline 10 Assista a aula de papel branco na. & 7 Confiança em minha alma cai dentro de \\
\hline 4 Quarto golpe de estado e o campo & 9 Grande general chega já e não creias \\
\hline 5 Sobre minha cabeça está de Deus pai & 10 Assista a aula de papel branco na. \\
\hline 3 Gosta muito crer te dá muito para & 6 Ação humilde é bem claro o céu \\
\hline
\end{tabular}


Anexo 2. Protocolo de marcação do teste de identificação de sentenças dicóticas (DSI) e lista para conferência do examinador

\begin{tabular}{|c|c|c|c|c|c|c|c|c|c|c|c|}
\hline \multicolumn{2}{|l|}{ Etapa / Item } & 2 & 3 & 4 & 5 & 6 & 7 & 8 & 9 & 10 & $\%$ acertos \\
\hline \multicolumn{12}{|l|}{ Treino OD } \\
\hline \multicolumn{12}{|l|}{ Treino OE } \\
\hline \multicolumn{12}{|c|}{ Integração OD } \\
\hline \multicolumn{12}{|c|}{ Integração OE } \\
\hline \multicolumn{12}{|c|}{ EDD } \\
\hline \multicolumn{12}{|l|}{ EDE } \\
\hline \multicolumn{12}{|c|}{ Faixa 2 - Treino } \\
\hline Item & \multicolumn{5}{|c|}{ Canal 1 - Orelha Direita } & & \multicolumn{5}{|c|}{ Canal 2 - Orelha Esquerda } \\
\hline 1 & \multicolumn{5}{|c|}{ Que ignora o fim principal é ganhar } & & \multicolumn{5}{|c|}{ A porta larga para ser mais rápido } \\
\hline 2 & \multicolumn{5}{|c|}{ Sobre minha cabeça está de Deus pai } & & \multicolumn{5}{|c|}{ Confiança em minha alma cai dentro de } \\
\hline 3 & \multicolumn{5}{|c|}{ Ação humilde é bem claro o céu } & & \multicolumn{5}{|c|}{ Sempre e corre muito mais bonito que } \\
\hline 4 & \multicolumn{5}{|c|}{ Grande general chega já e não creias } & & \multicolumn{5}{|c|}{ Assista a aula de papel branco na. } \\
\hline 5 & Gosta mu & er te & muito & ara & & & Quart & pe & & $\mathrm{cat}$ & \\
\hline Faixa $3-\operatorname{lnt}$ & gração Bin & & & & & & & & & & \\
\hline Item & Canal 1 - & רa D & & & & & Canal & Dre & Esqu & & \\
\hline 1 & Gosta mu & er te & muito & & & & Assist & aula & pap & anco & \\
\hline 2 & Ação hun & é be & laro o & & & & Grano & ner & nega & não & eias \\
\hline 3 & Sobre mi & $\mathrm{abec}$ & stá de & eus pai & & & Que is & 90 & oring & é ga & \\
\hline 4 & Grande $\mathrm{g}$ & $\mathrm{alch}$ & já e $n$ & creias & & & Semp & cor & uito & bor & que \\
\hline 5 & A porta la & ara & mais & ido & & & Sobre & ha & ça $\epsilon$ & de D & pai \\
\hline 6 & Confianç & $\operatorname{minh}$ & Ima c & dentro de & & & Ação & ilde & & o cé & \\
\hline 7 & Quarto gc & le es & o e o & mpo & & & A port & ga & ser & ráp & \\
\hline 8 & Que igno & $\mathrm{m} \mathrm{pl}$ & pal é & inhar & & & Gosta & to $\mathrm{C}$ & e dá & to $p$ & \\
\hline 9 & Sempre $\mathrm{e}$ & $\mathrm{mu}$ & mais & nito que & & & Confic & em & ha & cai & tro de \\
\hline 10 & Assista a & de $p$ & bran & na. & & & Quart & pe & stac & $\mathrm{ca}$ & \\
\hline Faixa 4 - Es & Ata Direcio & à D & & & & & & & & & \\
\hline Item & Canal 1 - & a D & & & & & Canal & Ore & Esqu & & \\
\hline 1 & Confianç & $\operatorname{minh}$ & Ima c & dentro de & & & Grano & ner & nege & não & eias \\
\hline 2 & Assista a & de $p$ & bran & na. & & & Semp & cor & uito & bor & que \\
\hline 3 & A porta la & ara & mais & oido & & & Confic & $\mathrm{em}$ & ha & cai & tro de \\
\hline 4 & Ação hun & é be & laro o & & & & Sobre & ha & ça $\epsilon$ & de D & s pai \\
\hline 5 & Sempre $\mathrm{e}$ & $\mathrm{e} \mathrm{mu}$ & mais & nito que & & & Que is & 90 & oring & é ga & \\
\hline 6 & Grande g & al ch & já e $n$ & creias & & & Gosta & to $\mathrm{C}$ & e dá & to $\mathrm{pe}$ & \\
\hline 7 & Que igno & $\mathrm{im} \mathrm{p}$ & pal é & anhar & & & Assist & aula & pap & anco & \\
\hline 8 & Quarto gc & de es & o e o & mpo & & & Ação & ilde & $\mathrm{em} \mathrm{c}$ & o cé & \\
\hline 9 & Gosta mu & er te & muito & ara & & & A port & ga & ser & ráp & \\
\hline 10 & Sobre mir & abec & stá de & Jeus pai & & & Quart & pe & stac & $\mathrm{ca}$ & \\
\hline Faixa 5 - Es & tta Direcio & à $\mathrm{E}$ & erda & & & & & & & & \\
\hline Item & Canal 1 - & ha D & & & & & Canal & Dre & Esqu & & \\
\hline 1 & Grande $\mathrm{g}$ & al ch & já e $n$ & o creias & & & Que is & 90 & ring & é ga & \\
\hline 2 & Ação hun & é be & laro o & & & & Assist & aula & pap & anco & \\
\hline 3 & A porta la & Dara & mais & pido & & & Grano & ner & nege & não & eias \\
\hline 4 & Sobre mir & abec & stá de & Jeus pai & & & Semp & cor & uito & bor & que \\
\hline 5 & Confianç & $\operatorname{minh}$ & Ima c & dentro de & & & Gosta & to $\mathrm{C}$ & e dá & to $\mathrm{pe}$ & \\
\hline 6 & Assista a & de $p$ & bran & na & & & A port & ga & ser & ráp & \\
\hline 7 & Quarto g & de es & o e o & ampo & & & Confic & em & & cai c & tro de \\
\hline 8 & Que igno & $\mathrm{im} \mathrm{pr}$ & pal é & anhar & & & Ação & ilde & $\mathrm{emc}$ & o cé & \\
\hline 9 & Sempre $\Theta$ & $\mathrm{e} \mathrm{mu}$ & mais & nito que & & & Quart & Ipe & stac & $\mathrm{oca}$ & \\
\hline 10 & Gosta mu & er te & muito & ara & & & Sobre & nha & eça $\epsilon$ & de D & s pai \\
\hline
\end{tabular}

Legenda: $\mathrm{OD}$ = orelha direita; $\mathrm{OE}$ = orelha esquerda; $\mathrm{EDD}$ = escuta direcionada à direita; $\mathrm{EDE}$ = escuta direcionada à esquerda 